\title{
On the Complexity of Exact Maximum-Likelihood Decoding for Asymptotically Good Low Density Parity Check Codes: A New Perspective
}

\author{
Weiyu Xu and Babak Hassibi \\ EE Department \\ California Institute of Technology \\ Pasadena, CA 91125 \\ weiyu,hassibi@caltech.edu
}

\begin{abstract}
The problem of exact maximum-likelihood (ML) decoding of general linear codes is well-known to be NP-hard. In this paper, we show that exact ML decoding of a class of asymptotically good low density parity check codes-expander codes-over binary symmetric channels (BSCs) is possible with an average-case polynomial complexity. This offers a new way of looking at the complexity issue of exact ML decoding for communication systems where the randomness in channel plays a fundamental central role. More precisely, for any bit-flipping probability $p$ in a nontrivial range, there exists a rate region of non-zero support and a family of asymptotically good codes which achieve error probability exponentially decaying in coding length $n$ while admitting exact ML decoding in average-case polynomial time.As $p$ approaches zero, this rate region approaches the Shannon channel capacity region. Similar results can be extended to AWGN channels, suggesting it may be feasible to eliminate the error floor phenomenon associated with belief-propagation decoding of LDPC codes in the high SNR regime. The derivations are based on a hierarchy of ML certificate decoding algorithms adaptive to the channel realization.In this process, we propose an efficient $O\left(n^{2}\right)$ new ML certificate algorithm based on the maxflow algorithm. Moreover, exact ML decoding of the considered class of codes constructed from LDPC codes with regular left degree, of which the considered expander codes are a special case, remains NP-hard; thus giving an interesting contrast between the worst-case and average-case complexities.
\end{abstract}

\section{INTRODUCTION}

ML decoding is a central algorithmic problem in coding theory[1], [2] since ML decoders minimize the message error probability when each codeword is transmitted with equal probability. For general linear block codes over binary symmetric channels (BSCs) the problem is as follows: given an $n \times m$ matrix $\mathbf{H}$ over $F_{2}$, a target vector $\mathbf{y} \in F_{2}^{m}$, and an integer $w>0$, is there a vector $\mathbf{v} \in F_{2}^{n}$ of weight $\leq w$, such that $\mathbf{v}^{t} \mathbf{H}=\mathbf{y}^{t}$ ? Berlekamp, McEliece, and van Tilborg [1] have shown that this problem is NP-hard using a reduction from the 3-dimensional matching problem. Since the publication of [1], the worst-case computational complexity of ML decoding of general linear codes has been extensively studied. The problem remains NP-hard even if the code is known in advance and can be preprocessed as long as desired[6]. The NP hardness also holds when it comes to some specific family of codes [2].

To quote further from [2], however, “...there is no nontrivial useful family of codes for which a polynomial-time maximum- likelihood decoding algorithm is known (such a result would, in fact, be regarded a breakthrough)". Also, the existing results are either for codes which are not asymptotically good or apply to too general a class of codes. ${ }^{1}$ On one hand, these results seem very discouraging for people to implement the ML decoding algorithm to achieve the best decoding performance. But on the other hand, these NP-hardness results are about the worst-case complexity from the viewpoint of pure computer science and in many applications such as in real communication systems, the worst-case complexity results may not be very useful because they can not fully capture the channel randomness which fundamentally characterizes the communications systems. In this paper, we will study the average-case complexity limit of the exact ML decoding for a specific class of asymptotically good codes taking into account the randomness in communications channels. Before doing that, we would try to state this problem more precisely and further clarify the motivations for and consequences of studying this type of complexity limit.

The randomness inherent in noisy communication channels imposes a fundamental limit on the achievable transmission performance and capacity, as explored and answered by Shannon's channel capacity theorem. However, the influence of the channel randomness on the complexity of exact ML decoding has long been neglected, although complexity is another central theme in designing communications systems[3]. The worst-case NP-hardness of the ML decoding is obtained over the problem space of a broad class of linear codes and all the possible channel realizations. But in the practical systems, the complexity of decoding algorithms might change substantially with different channel realizations. In fact, the hard input instances for a carefully designed ML decoder may occur with much less or even negligible probability compared with the easy instances. If the probability of the hard instances occurring is essentially zero (compared with the ML decoding error probability), we virtually never go into the hardest instances for that optimized decoder. So on average, the ML performance can be achieved with relatively low complexity if

\footnotetext{
${ }^{1}$ For $i=1,2, \ldots$, let $C_{i}$ be an $\left(n_{i}, k_{i}, d_{i}\right)$ linear code over $F_{2}$. The infinite sequence of codes $C_{1}, C_{2}, \ldots$ is said to be asymptotically good if $n_{i} \rightarrow \infty$, $k_{i} / n_{i} \geq R$ and $d_{i} / n_{i} \geq \delta$ for some positive $R$ and $\delta$.
} 
there is an ML decoder which sufficiently adapts to the channel realizations and works efficiently on a prevailing portion of the channel outputs (of course, an ML decoder should have a much smaller worst-case complexity than exhaustive search although the worst cases essentially never occur. This is possible, as the examples below showed).

Amazingly, among the well-known decoding schemes, we can find many excellent examples of decoding algorithms (not necessarily ML decoding algorithms) which are characterized by a probabilistic distribution of decoding complexity. One prominent example is sequential decoders for the convolutional codes, where people are interested in the decoding complexity distribution and have showed that the mean number of computations is bounded if the code rate is smaller than the computational cutoff rate $R_{\text {comp }}$ [4]. Leveraging the low average-case complexity, the sequential decoders can achieve excellent performance with convolutional codes of large constraint length and provide an excellent performance and complexity tradeoff[3]. Besides the suboptimal sequential decoder, let us now look at the example of an efficient exact ML decoder when applied to a $(128,64)$ binary extended $\mathrm{BCH}$ codes in [5]. In all the 35000 examples simulated, the priorityfirst search ML decoding algorithm in [5] performs nearly fifteen orders of magnitude more efficient on average than the exhaustive exact ML decoding using Wolf's algorithm [5]. Even in the worst case seen of all the simulation examples, its complexity is still of 10 orders of magnitude less. Clearly ML decoding is not so hopeless over the randomness of the channel, so we need more than worst-case complexity results to offer new insight in designing efficient exact ML algorithms. But we should notice that the sequential decoder is not an exact ML decoder and the ML decoder in [5] can not work efficiently under a fixed SNR when the coding length $n$ grows. Moreover, the codes in the previous two examples are not asymptotically good. Now an interesting question is, for a fixed bit flipping probability $p$ in BSC or fixed SNR in binary input AWGN (BI-AWGN) channels, whether we can have asymptotically efficient exact ML decoding algorithms for a family of asymptotically good codes as the coding length $n$ grows to infinity. If yes, under what data rates can we have such an efficient exact ML decoder? If such exact ML decoders exist, how can we design them?

The current paper deals with these issues; we show that a certain class of asymptotically good LDPCs (so-called expander codes) admit average-case polynomial-time ML decoding over BSCs and binary input AWGN (BI-AWGN) channels in certain rate regions, which strongly suggests the possibility of designing an efficient ML decoder for this family of codes, especially in the high SNR regime. (We are not saying that the problems discussed here belong to the class of EP (expected polynomial time) algorithmic problems, which are defined with respect to randomized algorithms[19].) This result is true regardless of whether preprocessing is applied to the code or not (Note without preprocessing,the expansion property of the Tanner graph is not available to the decoder in advance). Also the derivation is based on a hierarchy of ML certificate decoders with increasing complexity. By this we mean that, in some cases, the decoders can certify that the solution is the ML solution; thus, we either get an exact ML codeword or declare an error. Consider a series of $K$ such ML certificate decoders $D_{1}, D_{2}, \ldots, D_{K}$. Let $H_{i}$ be the set of received signal for which the ML certificate decoder $D_{i}, 1 \leq i \leq K$ can successfully find and certify the ML solution. We assume that $\Phi=H_{0} \subseteq H_{1} \subseteq H_{2} \subseteq \ldots H_{K-1} \subseteq H_{K}=\Xi$, where $\Phi$ is the empty set and $\Xi$ is the whole signal space. The receiver will start from $D_{1}$ and it will change from $D_{i}$ to $D_{i+1}$ for decoding if and only if the decoder fails by using $D_{i}$. Then the average-case complexity of the receiver is

$$
E N_{M L}=\sum_{i=1}^{K}\left(1-P\left(H_{i-1}\right)\right) N\left(D_{i}\right)
$$

, where $P\left(H_{i-1}\right)$ is the probability that the received signal falls in the signal space of $H_{i-1}$ and $N\left(D_{i}\right)$ is the complexity for performing decoder $D_{i}$. Here we consider the simplest cases with $K=2$ to show the existence of efficient ML decoders with average-case complexity. Let the code length be $n$, the rate $r$, the complexity of the suboptimal decoder $N(n)$, and the probability that the suboptimal decoder $D_{1}$ does not find the ML solution be $P_{e}(n)$. Then if we perform exhaustive search over the codebook whenever the suboptimal decoder fails to give an ML certificate, the expected complexity of the resulting ML algorithm, $E N_{M L}(n)$, will clearly be

$$
E N_{M L}(n)=N(n)+k(n) P_{e}(n) 2^{n R}
$$

where $k(n)$ is a polynomial constant representing the computation incurred per codeword in the exhaustive search. Clearly, if $P_{e}(n) 2^{n R} \rightarrow 0$ exponentially in $n$, then the ML algorithm will have expected polynomial-time complexity (equal to the complexity of the suboptimal decoder). Therefore in the remainder of the paper the main effort is to determine $\mathrm{ML}$ certificate decoders and to compute (or bound) $P_{e}(n)$.

One such ML certificate decoder is the linear programming (LP) decoder of Feldman [13]. We also propose a more efficient ML certificate decoder (reducing the worst-case complexity of the LP decoder from $O\left(n^{9}\right)$ to $O\left(n^{2}\right)$ ) which is based on the Ford-Fulkerson max-flow algorithm. We further characterize the achievable rate region $R_{M L}$ (albeit loosely) in which there exists a family of asymptotically good expander codes whose error probability goes to zero exponentially under an exact ML decoding algorithm with expected polynomial complexity. Finally, we observe that the argument in [1] can be used to show that exact ML decoding of the considered class of codes constructed from LDPC codes with regular left degree, of which the considered expander codes are a special case, remains NP-hard. This is reminiscent of the examples in [7] and many classical examples in computer science from references therein where the problems are NP-hard in the worst case but have a deterministic algorithm with average-case polynomial-time complexity over a practical input distribution.

These results potentially have practical implications for eliminating the error floor phenomenon associated with 
message-passage decoding of LDPC codes in the high SNR regime [8], [9], [10], [12]. Although the exact ML algorithms here are not practical by themselves, these results provide new insights into improving suboptimal BP algorithm in the high SNR regime by using a series of practical strengthening ML certificate decoders. One possible way is to use a series of LP decoders with tighter relaxations. But noticing that the original LP decoder in [13] is already very slow when compared with the BP decoder, the LP decoders may not be very good for this purpose. Actually, we are investigating LDPC decoding using another series of more efficient ML certificate algorithms and the initial experiment results are very encouraging[11]. In some sense, this paper theoretically verifies the possibility of achieving near-ML or even ML decoding performance by a series of strengthening efficient ML certificate decoders for asymptotically good LDPC codes.

This paper is organized as follows. Section II studies exact ML decoding for expander codes with expected polynomial complexity under unlimited preprocessing of the codes. Section III proposes a new ML certificate algorithm and shows that exact ML decoding in expected polynomial-time is possible even without preprocessing. The rate region $R_{M L}$ is also characterized in Section III. Section IV gives the brief proof of the NP-hardness of ML decoding of the considered class of codes constructed from LDPC codes with regular left degree.

\section{Average-Case Polynomial Complexity Exact \\ ML DECODING WITH UNLIMITED PREPROCESSING}

We begin by allowing for unlimited preprocessing of the codebook as it makes the problem simpler and sets the stage for the subsequent proofs. Thus, consider a BSC with bit flipping probability $0<p<\frac{1}{2}$. A Tanner graph $G$ is called a $(k, \Delta)$-expander if for every set $S$ of variable nodes where $|S| \leq k$, the number of check nodes incident to $S$ is larger than $\Delta|S|[14]$. We consider the family of binary parity-check leftregular expander graphs $G$ and its corresponding binary code $C^{\prime}$ [14] with $n$ coded bits, $m$ parity checks and rate at least $(1-m / n)$. Throughout the paper, $G$ has regular left degree $c$ for the variable nodes. We change the code $C^{\prime}$ by adding more parity check constraints to make a new code $C$ with length $n$ and rate $r \leq(1-m / n)$ when there exist dependent parity checks in $G$. Since $C$ is a subcode of $C^{\prime}$, we have

Lemma 1: The minimum distance $\mathbf{w}_{\min }$ of the new code $C$ of rate $r$ is no smaller than the minimum distance of the code $C^{\prime}$.

Pick a random codeword in $C$ for transmission and denote the received sequence by $\mathbf{r}$. Here is an ML certificate algorithm, where $d_{H}(\cdot, \cdot)$ denotes the Hamming distance.

\section{- Preprocessing}

1) Compute the minimum distance of the code $C$

\section{- Decoding}

1) If there is a variable that is in more unsatisfied than satisfied constraints (only the constraints in the expander graph $G$ ), flip the value of that variable

2) Repeat 1) until no such variable remains. Denote the resulting sequence as $\mathbf{x}^{\prime}$;
3) If $\mathbf{x}^{\prime}$ is in the code $C$ and $d_{H}\left(\mathbf{x}^{\prime}, \mathbf{r}\right) \leq \frac{\mathbf{w}_{\text {min }}}{2}$ declare $\mathbf{x}^{\prime}$ as the ML sequence. Otherwise, declare an error.

Step 1) and 2) in Decoding is basically the "simple sequential decoding" algorithm of [14]. The novelty here is that, if the minimum distance is known, an ML certificate can be provided. If we perform exhaustive search whenever the algorithm fails, we have an ML algorithm. To compute the probability of failure, $P_{e}(n)$, we need a lemma on the minimum relative distance (defined as $\mathbf{w}_{\min } / n$ ) of $C$.

Lemma 2: Let the bipartite expander graph $G$ be a $(\alpha n, 3 c / 4)$ expander, where $0<\alpha<1$ and $c$ is the constant left-degree. Then the minimum relative distance of $C$ is at least $3 \alpha / 2$.

Proof: Suppose that the minimum relative distance for the code $C^{\prime}$ is $\beta<3 \alpha / 2$. Then the set $\mathbf{S}$ of variable nodes having value ' 1 ' in a non-zero codeword with relative weight $\beta$ is connected to at least $\frac{2}{3} \times \beta n \times(3 c / 4)>(c / 2) \beta n$ parity check neighbors. Since each variable node can accommodate at most $c$ check nodes, there must be at lest one check node neighboring $\mathbf{S}$ that is connected to only one variable node in $\mathbf{S}$. But then that parity check can not be satisfied, a contradiction. Combining $\beta \geq 3 \alpha / 2$ with Lemma 1 , we get Lemma 2 .

Note: In Lemma 2, we assume $\alpha n, \beta n$ and $2 \beta n / 3$ are all integers, which for large enough $n$ is not an issue.

We can now compute $P_{e}(n)$.

Lemma 3: Let bipartite Tanner graph $G$ be an $(\alpha n, 3 c / 4)$ expander. Then $P_{e}(n)$ is upper bounded by by $2^{-D\left(\frac{\alpha}{2} \| p\right) n}$, where $\frac{\alpha}{2}>p$ and $D(x \| y)=x \log _{2} \frac{x}{y}+(1-x) \log _{2} \frac{1-x}{1-y}$ is the Kullback-Leibler divergence between Bernoulli random variables with parameters $x$ and $y$ respectively.

Proof: For the expander code $C^{\prime}$ corresponding to $G$, the simple sequential decoding algorithm can correct up to $\frac{\alpha}{2}$ fraction of errors in $\mathrm{O}(\mathrm{n})$ operations (Theorem 10 in [14]). Since $C$ is a subcode of $C^{\prime}$, the same is true of $C$. By Lemma 2 , the minimum relative distance for $C$ is at least $\frac{3 \alpha}{2}$. So if the fraction of errors is no larger than $\frac{\alpha}{2}$, the algorithm will provide the ML certificate. For $p<\frac{\alpha}{2}$, the probability of having no less than $\alpha / 2$ fraction of errors occurring is upper bounded by $2^{-D\left(\frac{\alpha}{2} \| p\right) n}$ from a standard Chernoff bound argument.

Lemma 4: For a fixed $0<x<1, D(x \| y)$ goes to infinity as $0<y<1$ approaches 0 .

Proof: Straightforward computation.

We can now give the main result of the section.

Theorem 1: Let Tanner graph $G$ be an $(\alpha n, 3 c / 4)$ expander. Then the expected computational time of an ML decoding algorithm over the BSC channel, $N_{M L}(n)$, is polynomial in $n$, given that $p<\frac{\alpha}{2}$ and $r<D\left(\frac{\alpha}{2} \| p\right)$. The storage complexity is kept polynomial in $n$ in the worst case.

Proof: The first statement follows from Lemma 3 and (2). For the second statement, we note that since each codeword is generated one by one the storage complexity is kept polynomial in $n$ in the worst case.

Rather than fixing $p$ and looking at the rate, we can fix the rate $r$ and look at the flipping probability $p$.

Theorem 2: For any rate $0<r<1$, there exists a threshold 
$0<p^{*}<1$ and a family of asymptotically good block codes with rate $r$ and length $n$ such that when allowing preprocessing of the codes, exact ML decoding can be achieved with an expected polynomial complexity in $n$ over the BSC with bit flipping probability $0 \leq p<p^{*}$. Furthermore, the block error probability of this family of codes decreases exponentially in $n$ asymptotically.

Proof: For any rate $r$, with sufficiently large but constant left degree $c$ and right degree $d\left(r=1-\frac{c}{d}\right)$, there exists a number $\alpha>0$ and a family of expander graphs $(\alpha n, 3 c / 4)$ with the number of variable nodes $n$ going to infinity [16]. By Lemma 2, such expander graphs give a family of codes of length $n$ with minimum relative distance of $3 \alpha / 2$ and rate $r$. Since $\frac{\alpha}{2}>0$, by Lemma 4 , there must be a number $p^{*}$ so that for any $p<p^{*}$, we have $p<\frac{\alpha}{2}$ and $r<D\left(\frac{\alpha}{2} \| p\right)$. By Theorem 1, the first statement holds. For the second statement, since the ML decoder corrects up to $3 \alpha / 4$ fraction of errors, which is larger than $p$, we have an exponentially decreasing error probability from the Chernoff bound.

Lemma 5: For any family of $(\alpha n, 3 c / 4)$ expander codes with increasing code length $n$ and a constant $\alpha>0$, the improvement in the lower bound of the error exponent by using the ML algorithm instead of the "simple sequential decoding algorithm" [14] for this family of codes is arbitrarily large if $p$ is sufficiently small but remains positive. However, this improvement comes with expected polynomial complexity in $n$ when allowing preprocessing of the codes.

Proof: By using the Chernoff bound, the block error rate of ML decoding is upper bounded by $2^{-D\left(\frac{3 \alpha}{4} \| p\right) n}$, while the block error rate of iterative decoding is upper bounded by $2^{-D\left(\frac{\alpha}{2} \| p\right) n}$ when $p<\frac{\alpha}{2}$. Since $D\left(\frac{3 \alpha}{4} \| p\right)-D\left(\frac{\alpha}{2} \| p\right) \geq$ $\frac{\alpha}{4} \log _{2}\left(\frac{3 \alpha}{4} / p\right)+c^{\prime}$, where $c^{\prime}$ is a constant, this difference grows to infinity as $p \rightarrow 0$.

We now briefly consider BI-AWGN channels while allowing preprocessings of the codes. To do ML decoding, we first make a hard decision on the received sequence $\mathbf{r}^{\prime}$. Then we send the hard-decided sequence $\mathbf{r}$ to an exact ML decoder as in BSC channels except we change the Hamming distance to the Euclidean distance. Using a union bound, the $P_{e}(n)$ is upper bounded by $P_{1}+P_{2}$, where $P_{1}$ is the probability of more than $\alpha / 2$ bit errors occurring in $\mathbf{r}$ and $P_{2}$ is the probability that the noise vector's $l_{2}$ norm is larger than one half of the minimum Euclidean distance between codewords. By the Chernoff bound for Bernoulli and chi-square random variables (details omitted) and Lemma 2 , we have $P_{1} \leq 2^{-D\left(\frac{\alpha}{2} \| P_{\text {err }}\right) n}$ and $P_{2} \leq e^{-\frac{1}{2}\left(\frac{3}{2} S N R-1-\ln \left(\frac{3}{2} S N R\right)\right) n}$, where $\bar{P}_{\text {err }}$ is the hard decision bit error probability for BI-AWGN channels, which improves as the SNR increases. Using the same arguments as for the BSC, we have counterparts to Theorems 1 and 2 for BI-AWGN channels when the SNR is high enough.

In this section, the key to obtaining a polynomial-time ML certificate algorithm was knowing the minimum distance of the code (which must be precomputed). We now show that such ML certificate algorithms can be obtained without preprocessing.

\section{Average-Case Polynomial Complexity Exact ML DECODING WITHOUT PREPROCESSING}

In LP decoding over memoryless channels the problem is relaxed to a linear programming problem with polynomial complexity [13]. The LP decoder has the ML certificate property: if the solution to the relaxed LP is integral it is the ML codeword. The reader is referred to [13] for further details.In [15], by constructing a feasible point for the dual of the LP, the authors prove that the LP decoder can correct a constant fraction of errors when applied to expander codes of sufficient expansion, which is stated in the following theorem:

Theorem 3: [15]Let $C$ be a low-density parity-check code with length $n$ and rate at least $1-m / n$ described by a Tanner graph $G$ with $n$ variable nodes, $m$ check nodes, and regular left degree $c$. Suppose $G$ is an $(\alpha n, \delta c)$-expander, where $\delta>2 / 3+$ $1 /(3 c)$ and $\delta c$ is an integer. Then the LP decoder succeeds and gives the ML solution, as long as at most $\frac{3 \delta-2}{2 \delta-1}(\alpha n-1)$ bits are flipped by the channel.

The above theorem implies that $P_{e}(n)$ can be computed as in the previous section and that the LP decoder, in conjunction with exhaustive search, is an expected polynomial time ML decoder. However, the main disadvantage of LP decoding is the complexity coming from solving a linear program. As noted in [13], the worst-case total number of variables and constraints in the LP relaxation is of order $O\left(n^{3}\right)$ if we consider an expander graph with irregular check degrees, where $n$ is the length of the codes. To solve a general LP, the complexity is of order $O\left(\mathrm{dim}^{3}\right)$, where $\mathrm{dim}$ is the number of variables and constraints. This implies a complexity of order $O\left(n^{9}\right)$. We now show that the ML certificate property can be achieved with worst-case complexity $O\left(n^{2}\right)$, without sacrificing the guaranteed performance.

\section{New ML Certificate Algorithm}

1) If there is a variable that is in more unsatisfied than satisfied constraints (only the constraints in the expander graph), flip the value of that variable

2) Repeat 1) until no such variable remains. Denote the resulting sequence as $\mathbf{x}^{\prime}$.

3) If $\mathbf{x}^{\prime}$ is not in the code $C$, go to 4). Otherwise,construct a series of max-flow instances as follows: Introduce a source node $s$ and a sink node $t$. Let $U$ denote the set of variables nodes where $\mathbf{r}$ and $\mathbf{x}^{\prime}$ differ, $N(U)$ denote the check node neighborhood of $U$ and let $\tilde{U}$ denote the set of variable nodes other than $U$ that are connected to $N(U)$. Take an integer $A=\left\lfloor\frac{c}{2}\right\rfloor+1$. Add directed edges from $s$ to the set of variables $U+\tilde{U}$. If $i \in U$, assign capacity $A$ to the directed edge from $s$ to $i$. For each $i \in \tilde{U}$, if $|N(i) \bigcap N(U)|>(2 A-c)$, assign integer capacity $|N(i) \bigcap N(U)|-(2 A-c)$ to the directed edge from $s$ to $i$, otherwise assign capacity 0 to it. Construct directed edges from any $i \in U \cup \tilde{U}$ to its neighbors in $N(U)$ and assign integer capacity 1 to them. Construct directed edges from each check node in $N(U)$ to the sink node $t$ and assign capacity 1 to them.

Use the Ford-Fulkerson algorithm to find the max-flow from the source $s$ to the sink $t$. If the max-flow value 
is equal to the sum of the capacities of the edges from $s$ to $U \cup \tilde{U}$, then declare $\mathbf{x}^{\prime}$ as the ML sequence. If this does not hold but $A<c$, increase $A$ by 1 and construct a new max-flow instance. Otherwise, go to 4).

4) Declare an error.

When the capacities are integers the runtime of FordFulkerson algorithm is bounded by $O(E * f)$, where $E$ is the number of edges in the graph and $f$ is the maximum flow in the graph [18]. $E$ is of order $O(n)$ since each variable node has a constant degree $c$ and $f$ is also of order $O(n)$ since it is upper bounded by $\mathrm{cn}$. Obviously the max-flow algorithms are performed at most $c$ times. Since each step of the new ML certificate algorithm is of order $O\left(n^{2}\right)$, the total complexity is of order $O\left(n^{2}\right)$.

Lemma 6: If the max-flow attains the sum of edge capacities from the source $s$ to $U \cup \tilde{U}$ for some $A \geq\left\lfloor\frac{c}{2}\right\rfloor+1$, then $\mathbf{x}^{\prime}$ must be an exact ML codeword.

Proof: There is a maximum flow such that the flow through every edge is integral[18]. If the max-flow value is equal to the sum of edge capacities from $s$ to $U \cup \tilde{U}$, each node $i$ in $U$ is connected to a set $M(i)$ of $A$ parity check nodes $(M(i) \bigcap M(j)=\phi$ if $i \neq j)$. Suppose there is a codeword $\mathbf{x}^{\prime \prime}$ such that $d_{H}\left(\mathbf{x}^{\prime \prime}, \mathbf{r}\right)<d_{H}\left(\mathbf{x}^{\prime}, \mathbf{r}\right)$. Then $\mathbf{x}^{\prime \prime}$ must share the same values with $\mathbf{r}$ in $l>0$ positions of $U$ (otherwise, $\left.d_{H}\left(\mathbf{x}^{\prime \prime}, \mathbf{r}\right) \geq d_{H}\left(\mathbf{x}^{\prime}, \mathbf{r}\right)\right)$. We show that outside $U, \mathbf{x}^{\prime \prime}$ must be different from $\mathbf{r}$ in at least $l$ places, which contradicts $d_{H}\left(\mathbf{x}^{\prime \prime}, \mathbf{r}\right)<d_{H}\left(\mathbf{x}^{\prime}, \mathbf{r}\right)$. Without loss of generality, we assume that $\mathbf{x}^{\prime}$ is the all-zero codeword. Then the $l$ shared bits (denoted by the set $W \subseteq U$ ) in $U$ by $\mathbf{x}^{\prime \prime}$ and $\mathbf{r}$ are $l$ ' 1 's. Obviously, the bit set $W$ has $A \times l$ neighbor check nodes induced by the max-flow instance (we denote these $A l$ neighbor check nodes by the set $N^{\prime}(W)$ ). For each check node in $N^{\prime}(W)$, at least 2 variable nodes are of value ' 1 ' to make $\mathbf{x}^{\prime \prime}$ a codeword. So besides the $A l$ ' 1 's provided by the set $W$ through the active flow edges in the max-flow instance, we need another $B$ ' 1 's to satisfy the parity checks in $N^{\prime}(W)$, where $B \geq$ $2 A l-A l=A l$. However, the $l$ bits in $W$ can provide at most $(c-A) \times l$ extra ' 1 's. Thus, for $\mathbf{x}^{\prime \prime}$ we must have at least $B-(c-A) \times l \geq A \times l-(c-A) \times l=(2 A-c) \times l$ edges which are connected to $N^{\prime}(W)$ but emanate from variable nodes of value ' 1 ' outside $U$. From the construction of the max-flow instance, each variable node outside $U$ can contribute at most $(2 A-c)$ ' 1 's to $N^{\prime}(W)$. So $\mathbf{x}^{\prime \prime}$ has at least $l$ variable nodes of value ' 1 ' outside $U$, a contradiction since $\mathbf{r}$ is all-zero outside $U$.

Although this new algorithm uses max-flow arguments as in the analysis of the LP decoder [15], there are several key differences. The max-flow argument in [15] is for the purpose of analysis in proving the existence of a dual feasible point, but in the new algorithm max-flow arguments are used directly in the computation, thus reducing the complexity by avoiding solving a large linear program. The series of max-flow instances here are much more refined because they use optimized rather than uniform link capacities from the source to the variable nodes in the max-flow instance of [15]. Moreover, without looking for the dual feasible edge weight assignment, the new algorithm and its direct proof provide more intuition about why expander codes efficiently correct a constant fraction of errors while having the ML certificate property even without preprocessing. The Ford-Fulkerson algorithm can be easily integrated into any belief propagation decoder to efficiently offer them the ML certificate property, which can help the decoder decide whether it is necessary to perform more computations to improve the performance. The following lemma gives a performance guarantee of the new ML certificate algorithm.

Lemma 7: Suppose $\delta>2 / 3+1 /(3 c)$, and $\delta c$ is an integer. Then if $\mathbf{x}^{\prime}$ is in the code $C$ and is different from $\mathbf{r}$ in at most $\frac{3 \delta-2}{2 \delta-1}(\alpha n-1)$ positions, $\mathbf{x}^{\prime}$ will be certified to be an ML codeword in the new ML certificate algorithm. When $\delta=3 / 4$, the new algorithm is guaranteed to correct up to $\alpha / 2$ fraction of errors while providing ML certificate property, which matches the proved capability of LP decoder.

Proof: In [15] the authors showed the conditions in this lemma imply the existence of a $\delta$-matching of $U$ (see the definition in [15]). We show that the number of edges connected to each variable node $i$ in a $\delta$-matching of $U$ is no smaller than the capacity from the source $s$ to $i$ for $A=\delta c$, thus implying a maximum flow as specified in Lemma 6. As in [15], define $\dot{U}=\{i \in V: i \notin U,|N(i) \bigcap N(U)| \geq$ $(1-\lambda) c+1\}$ and let $\lambda=2(1-\delta)+1 / c$. If $i \in U$, the number of connected edges in $\delta$-matching is equal to the the capacity $A$. If $i \notin U \cup \dot{U}$, the capacity from $s$ to $i$ is zero because $(1-\lambda) c-(2 A-c)=-1<0$. If the variable node $i \in \dot{U}$, let $B$ be the number of check nodes among $N(U)$ that $i$ is connected to by the $\delta$-matching. From the definition of $\delta$-matching, the node $i$ will be incident to at least $\lambda c$ edges in the $\delta$-matching. Suppose $B<|N(i) \bigcap N(U)|-(2 A-c), i$ is incident to less than $|N(i) \bigcap N(U)|-(2 A-c)+|N(i) \bigcap \overline{N(U)}|=$ $|N(i)|-(2 A-c)=2 c(1-\delta)<\lambda c$ edges in the $\delta$-matching, a contradiction. Thus there will be maximum flow satisfying Lemma 6 since we can assign the flow from the source to the edges in the $\delta$-matching. Since by the step 1) and step 2) we can correct up to $\alpha / 2$ fraction of errors for an expander graph with $\delta=3 / 4$. Also, the maximum flow instances provide the exact ML sequence.

From Lemma 6 and 7, we see that the new ML certificate algorithm corrects a constant fraction of errors with low complexity. Since the LP decoder and the newly proposed ML certificate decoder can correct a constant fraction of errors without preprocessing, we have a counterpart of Theorem 2 for the case of no preprocessing allowed.

For a BSC channel with fixed bit flipping probability $p$, let us denote $R_{M L}(p)$ as the set of rates $t$ in which there exists a family of asymptotically good codes whose error probability goes to zero exponentially in the coding length under an expected polynomial complexity exact ML decoding algorithm without preprocessing. We now give an achievable region of $R_{M L}(p)$, for $0<p<1 / 2$. Clearly, $R_{M L}(p)$ has the channel capacity $1-H(p)$ as an upper bound.

Lemma 8: For a fixed $0<p<1 / 2$, the rate set $R(p) \subseteq$ $R_{M L}(p)$, where $R(p)$ is the set of rates $t$ such that $t \leq$ $r, p<\frac{3 \delta-2}{2 \delta-1} \alpha, t<D\left(\frac{3 \delta-2}{2 \delta-1} \alpha \| p\right)$, where $\alpha=\left(2 e^{\delta c+1}(\delta c /(1-\right.$ 
$\left.r))^{(1-\delta) c}\right)^{-\frac{1}{(1-\delta) c-1}}$, for some $r, c, \delta$ satisfying $0<r<1, c \in$ $N,(2 / 3+1 /(3 c))<\delta<1, \delta c \in N,(1-\delta) c \geq 2$.

Proof: It can be shown using random graphs that for the $r, \delta$ and $c$ in Lemma 8, there is a bipartite graph $G$ for any $n$ variable nodes and $(1-r) n$ check nodes, which is an expander $(\alpha n, \delta c)[15]$. By constructing a linear code with rate $t$ from the expander graph (noticing that the rate of the code $t$ can be made smaller than $r$ ) and applying the expected polynomialtime ML decoders described, we get the desired result.

Lemma 9: If $p$ is sufficiently close to zero but remains positive, the gap between the channel capacity and the supremum of the rate region $R_{M L}(p)$ is arbitrarily small.

Proof: Take any $0<\epsilon<1$,let $r=1-\epsilon, t=r$ and choose any $c$ and $\delta$ according to Lemma 8. Then from Lemma 8 ,there is a $p^{*}$ such that for $0<p<p^{*}$, such that $t=r \in$ $R_{M L}(p)$. But the channel capacity for any $p$ in the region $0<p<p^{*}$ is at most 1 , which is no bigger than $t+\epsilon$.

\section{Average-CAse Complexity versus Worse-CAse COMPLEXITY:A CONTRAST}

In this part, we prove that for any $0<t<1, t \leq r<$ 1 , the exact ML decoding problem remains NP-hard for the family of codes $C$ of rate $R \leq t$ constructed by adding linear constraints to the $L D P C$ codes $C^{\prime}$ of rate at least $r$ defined by Tanner graphs $G$ with regular left degree $c \geq 3$. This family of codes correspond to the codes we discussed in the previous two sections with the difference that we do not require the Tanner graph to be an expander graph.

Let the newly added linear constraints, the linear constraints corresponding to the check nodes in $G$ and the syndrome $\mathbf{y}$ be revealed to the decoder.We show that even in this more restricted case, the ML decoding problem is NP-hard.

Proof: Our proof essentially follows that of [1]. As long as the flipping probability $0<p<1$, the received codeword can be any binary sequence of length $n$. We reduce the $k$ dimensional matching problem (k-DM) to the ML decoding problem, where $k=c$. It is known that the $\mathrm{k}$-dimensional matching problem is NP-hard for $k \geq 3$ [17]. The decision problem for the $c$-dimensional matching problem is as follows: given a subset $U \subseteq T \times T \cdots \times T$, where $T$ is a finite set and the elements of $U$ are c-tuples from the set $T$, determine whether there is a set $W \subseteq U$ such that $|W|=|T|$, and no two elements of $W$ agree in any coordinate.

Without loss of generality, we assume that the cardinality of $U$ is larger than $|T|$, otherwise the corresponding c-dimensional matching problem will be trivial. Just as in [1], for any such c-DM problem, encode the set $U$ of ctuples into a $|U| \times c|T|$ incidence matrix $M$, in which each row corresponds to one of the $c$-tuples and has weight $c$, with each 1 corresponding to a component of the c-tuple. Sequentially repeat each row of the binary incidence matrix by $I=\max \left\{\left\lceil\frac{c|T|}{r|U|}\right\rceil,\left\lceil\frac{1}{t}\right\rceil\right\}$ times to create a new binary incidence matrix $M^{\prime}$ of size $(I|U|) \times(c|T|)$, where every consecutive $I$ rows are the same. Since $|U|>|T|$ and $t$ is fixed, $I$ is upperbounded by a constant.Let the code corresponding to $M^{\prime}$ be $C^{\prime}$. Let the added $(I-1)|U|$ linear constraints be the simple constraints which specify the $(I-1)|U|$ bits corresponding to the duplicate copies in $M$ of any row in $M^{\prime}$ to be zero. Combine these $(I-1)|U|$ simple constraints with $M^{\prime}$, we get a new parity check matrix $M^{\prime \prime}$ of dimension $I|U| \times((I-1)|U|+$ $c|T|)$. Thus the new code $C$ corresponding to the new parity check matrix $M^{\prime \prime}$ is a valid example in the considered family of codes. Take $\mathbf{y}=(0,0,0, \ldots .0,1,1, \ldots, 1)^{T}$, where $\mathbf{y}$ has $(I-1) \times|U| 0$ 's and $c|T| 1$ 's. Suppose we have a polynomialtime algorithm for the ML decoding of the considered family of codes, we just run the putative ML decoding algorithm with the parity check matrix as $M^{\prime \prime}$, the syndrome as $\mathbf{y}$, and $w=|T|$, we will know the answer to the $k$-dimensional matching problem. So the ML decoding of the considered family of codes is NP-hard in the worst case.

\section{ACKNOWLEDGMENT}

We thank Professor R.J.McEliece for helpful discussions.

\section{REFERENCES}

[1] E.R. Berlekamp, R.J.McEliece, and H.C.A. Van Tilborg, "On the inherent intractability of certain coding problems," IEEE Transactions on Information Theory, vol. 24, issue. 5, pp. 384-386, May 1978;

[2] V.Guruswami,A.Vardy, "Maximum-likelihood decoding of Reed-Solomon codes is NP-hard," IEEE Transactions on Information Theory, vol. 51, issue. 7, pp. 2249-2256, 2005;

[3] J.D. Forney, "Performance and Complexity," Proceedings of IEEE International Symposium on Information Theory, 1995. pp. 17-22, 1995;

[4] F. Jelinek, "An Upper Bound on Moments of Sequential Decoding Effort." IEEE Transactions on Information Theory, vol. 15, pp. 140-149,1969;

[5] Y.S.Han,C.R.P. Hartmann, Chih-Chieh Chen,"Efficient priority-first search maximum-likelihood soft-decision decoding of linear block codes," IEEE Transactions on Information Theory, vol. 39, vol. 39,pp. 1514 1523,1993;

[6] J. Bruck and M. Naor, "The hardness of decoding linear codes with preprocessing," IEEE Transactions on Information Theory, vol. 36, pp. 381385, March 1990;

[7] M. Krivelevich and D. Vilenchik, "Solving random satisfiable 3CNF formulas in expected polynomial time," Proceedings of the 17th Symposium on Discrete Algorithms, pp. 454-463,2006;

[8] T.Richardson, "Error Floors of LDPC codes", in Proceedings of 41st Allerton Conference on Communications, Computing and Control,2003;

[9] X. Huang, "Near perfect decoding of LDPC codes," in Proceedings of International Symposium on Information Theory 2005, pp.302-306, 2005;

[10] Marc P.C. Fossorier,"Iterative Reliability-Based Decoding of LowDensity Parity Check Codes," in IEEE Journal on Selected Areas in Communications, vol. 19,pp.908-917 May 2001.

[11] Weiyu Xu,"Efficient ML decoding of LDPC codes," in preparation;

[12] Pascal O. Vontobel and Ralf Koetter,"Graph-Cover Decoding and FiniteLength Analysis of Message-Passing Iterative Decoding of LDPC Codes," submitted to IEEE Transactions on Information Theory;

[13] Jon Feldman, Martin Wainwright and D. R. Karger, "Using linear programming to Decode Binary linear codes,"IEEE Transactions on Information Theory, vol. 51, pp. 954-972, 2005;

[14] Michael Sipser and Daniel A.Spielman, "Expander Codes", IEEE Transactions on Information Theory, Vol 42, No 6, pp. 1710-1722,1996;

[15] Jon Feldman, Tal Malkin, Cliff Stein, Rocco A. Servedio, Martin J. Wainwright,"LP Decoding Corrects a Constant Fraction of Errors", IEEE Transactions on Information Theory,vol.53,No.1,pp.82-89, 2007;

[16] D.Burshtein and G.Miller. "Expander Graph arguments for messagepassing algorithms", IEEE Transaction on Theory, 782-790,February 2002.

[17] C.Papadimitrou, Computational Complexity,Addison Wesley,1994

[18] Thomas H. Cormen, Charles E. Leiserson, Ronald L. Rivest, and Clifford Stein. Introduction to Algorithms, Second Edition, pp.651-664, MIT Press and McGraw-Hill, 2001.

[19] Ingo Wegener, Complexity Theory, Springer, Heidelberg, 2005. 